\title{
Editorial
}

\section{Understanding pain and posttraumatic stress disorder comorbidity: Do pathological responses to trauma alter the perception of pain?}

PTSD develops following exposure to a situation or event that is, or is perceived to be, threatening to the safety or physical integrity of one's self or others. Approximately $80 \%$ of the general Canadian and US population report having experienced an event of this type, and approximately $7 \%$ to $12 \%$ develop PTSD $[4,15]$. Prevalence is typically much higher in populations at risk for trauma exposure (e.g., deployed military personnel, [1]). Recent research indicates that PTSD is frequently accompanied by acute pain episodes as well as chronic musculoskeletal pain $[2,13,16]$. The co-occurrence of PTSD and chronic pain amplifies the negative effects of each, often complicating the treatment and resulting in poor outcome [3].

In this issue, Defrin et al. evaluated thresholds to noxious and non-noxious thermal and mechanical stimuli as well as pain intensity ratings of suprathreshold thermal stimuli in participants with PTSD (most of whom had chronic pain), a clinical control group with an anxiety disorder other than PTSD, and healthy controls. The general finding was that those with PTSD had significantly higher thermal detection and pain thresholds (lower for cold thresholds) than did control participants, but they reported suprathreshold pain stimuli as much more intense than did control participants. The former finding suggests that PTSD may be associated with hyposensitivity to non-noxious stimuli and hypoalgesia (i.e., decreased sensitivity) to at-pain threshold noxious stimuli, whereas the latter finding suggests hyperalgesia (i.e., increased pain responsiveness) to stimuli above the threshold for pain. These findings are in contrast to the hyperalgesia and hyperreactivity to pain stimuli typically observed in patients with chronic pain $[2,12]$. Defrin et al. offer two explanations for these findings, including altered attentional (e.g., attentional biases, dissociation) and sensory processing, although notably, neither acknowledges the confounding of pain and PTSD in the PTSD sample.

The strategy employed by Defrin et al. holds promise for determining whether pathological responses to traumatic experiences (e.g., PTSD) somehow alter pain perception and thereby serve as a mechanism that facilitates the development of chronic pain. However, this area is replete with complexities. There is, for example, evidence that hyperalgesia is induced by elevations in state and trait anxiety $[5,11]$. Elevated state and trait anxiety, neither of which was considered by Defrin et al., are characteristic of both PTSD and chronic pain; thus, it is plausible that PTSD and chronic pain serve to heighten sensitivity to noxious inputs and pain through the impact they have on anxiety. Conversely, there is evidence that conditioned stress-induced hypoalgesia/analgesia may play an important and potentially causal role in both PTSD $[9,14]$ and the behaviors that maintain chronic musculoskeletal pain $[7,8]$. The idea that decreased sensitivity to noxious stimuli is an important mechanism in PTSD and chronic musculoskeletal pain is somewhat counterintuitive; yet, the literature suggests that stress-related dysregulation of the endogenous opioid system - perhaps functioning to deactivate fear structures (e.g., amygdala hippocampus, thalamus) in the short term through heightened release of endogenous opioids - may play a role in blunting pain perception, reducing adaptive behavior, and increasing emotional numbing associated with these conditions. Thus, the findings of Defrin et al. regarding hypoalgesia may not be as "unexpected" [6] as they state. Indeed, Geuze et al. [10] recently reported that veterans with PTSD (without chronic pain), compared to veterans without PTSD, exhibited decreased sensitivity to fixed intensity heat pain stimuli which was associated with increased activation of the left hippocampus and decreased activation in the prefrontal cortex and right amygdala.

Defrin et al. highlight the importance of disentangling the complexities of co-occurring PTSD and chronic pain, and set the stage for further investigation of pain perception in traumatized individuals that may ultimately yield important data regarding underlying mechanisms. One noteworthy limitation of their study is that PTSD and pain were confounded (naturally so, perhaps), making it impossible to know whether the observed differences in pain threshold and magnitude 
estimation are a function of either or both disorders. It is, however, interesting to note that their results appear to be a hybrid of what has been found in PTSD patients (without chronic pain) and chronic pain patients (without PTSD); namely, hypoalgesia and a combination of hyperalgesia and hyperreactivity, respectively. Determination of the underlying mechanism or mechanisms awaits further investigation.

Along with Geuze et al. [10], Defrin et al. are the first to directly and systematically assess pain perception in participants with PTSD. Defrin et al. provide evidence of hyperalgesia to noxious stimuli but, as noted above, the method does not permit firm conclusions regarding the underlying mechanism. Collectively, these investigations provide fairly convincing evidence that PTSD is associated with pain hypoalgesia. However, because of methodological differences (e.g., different assessment strategies, inclusion of PTSD participants with and without chronic pain), it is difficult to draw firm conclusions regarding the potential role of altered pain perception in the co-occurrence of PTSD and chronic pain. These discrepancies point to the need of using a uniform method of quantitative sensory assessment to avoid problems of interpretation and to facilitate inter-study comparison. The magnitude estimation method used by Defrin et al. may not be ideal in that as betweengroup differences in pain threshold become larger, the heat pain stimulus exposure time increases differentially in the group with the higher threshold, raising the possibility that the increased pain ratings to suprathreshold stimuli may reflect, in part, longer exposure times.

To clarify these issues, further investigations are needed that account for (a) the role of anxiety and other dispositional constructs that impact pain perception and processing and (b) the unique contributions to the perception of pain from PTSD symptoms and current (and chronic) pain experiences. This might entail, for example, investigating the relationship between pain perception and traumatic experiences that involve personal injury (reported by $50 \%$ of PTSD participants in the Defrin et al. investigation) versus those involving witnessing a traumatic event (without personal injury). It is answers to these questions that may lead to improved management of the many individuals who experience co-occurring PTSD and chronic pain and, thereby, reduce their suffering and improve their quality of life.

\section{Acknowledgments}

G.J.G.A. is supported by a Canadian Institutes of Health Research (CIHR) Investigator Award. J.K. is supported by a CIHR Canada Research Chair in Health Psychology at York University. This work was funded in part by a CIHR New Emerging Team Grant \# PTS-63186. The authors have no conflicts of interest to declare.

\section{References}

[1] American Psychiatric Association. Diagnostic and statistical manual of mental disorders. 4th ed. (text revision; DSM-IV-TR). Washington, DC: American Psychiatric Association.

[2] Asmundson GJG, Coons MJ, Taylor S, Katz J. PTSD and the experience of pain: research and clinical implications of shared vulnerability and mutual maintenance models. Can J Psychiatry 2002;47:930-7.

[3] Asmundson GJG, Taylor S. PTSD and chronic pain: cognitivebehavioral perspectives and practical implications. In: Young G, Kane AW, Nicholson K, editors. Causality: Psychological knowledge and evidence in court. New York: Springer; 2006. p. $225-41$.

[4] Beckham JC, Davidson JRT, March JS. Anxiety disorders: traumatic stress disorders. In: Tasman A, Lieberman J, Kay J, editors. Psychiatry. London: Wiley; 2003. p. 1281-97.

[5] Carter LE, McNeil DW, Vowles KE, Sorrell JT, Turk CL, Ries BJ, et al. Effects of emotion on pain reports, tolerance and physiology. Pain Res Manage 2002;7:21-30.

[6] Defrin R, Ginzburg K, Solomon Z, Polad E, Bloch M, Govezensky $M$, Schreiber S. Quantitative testing of pain perception in subjects with PTSD-Implications for the mechanism of the coexistence between PTSD and chronic pain. Pain 2008;138:450-49.

[7] Flor H, Birbaumer N, Schulz R, Grüsser SM, Mucha RF. Pavlovian conditioning of opioid and nonopioid pain inhibitory mechanisms in humans. Eur J Pain 2002;6:395-402.

[8] Flor H, Grüsser S. Conditioned stress-induced analgesia in humans. Eur J Pain 1999;3:317-24.

[9] Foa EB, Zinbarg R, Rothbaum BO. Uncontrollability and unpredictability in post-traumatic stress disorder: an animal model. Psychol Bull 1992;112:218-38.

[10] Geuze E, Westenberg H, Jochims A, de Kloet CS, Bohus M, Vermetten $\mathrm{E}$, et al. Altered pain processing in veterans with posttraumatic stress disorder. Arch Gen Psychiatry 2007;64:76-85.

[11] James JE, Hardardottir D. Influence of attention focus and trait anxiety on tolerance of acute pain. $\mathrm{Br} \mathrm{J}$ Health Psychol 2002;7:149-62.

[12] Merskey H, Bogduk N. Classification of chronic pain, description of chronic pain syndromes and definitions of pain terms. Seattle: IASP Press; 1994.

[13] Otis JD, Keane TM, Kerns RD. An examination of the relationship between chronic pain and post-traumatic stress disorder. J Rehabil Res Dev 2003;40:397-406.

[14] Pitman RK, Orr SP, van der Kolk BA, Greenberg MS, Meyerhoff JL, Mougey EH. Analgesia: a new dependent variable for the biological study of posttraumatic stress disorder. In: Wolf ME, Mosnaim AD, editors. Posttraumatic stress disorder: Etiology, phenomenology, and treatment. Washington, DC: American Psychiatric Association; 1990, p. 141-7.

[15] Seedat S, Stein MB. Posttraumatic stress disorder: a review of recent findings. Curr Psychiatry Rep 2001;3:288-94.

[16] Shipherd JC, Keyes M, Jovanovic T, Ready DJ, Baltzall D, Worley V, et al. Veterans seeking treatment for posttraumatic stress disorder: what about comorbid chronic pain? J Rehabil Res Dev 2007;44:153-66. 
Gordon J.G. Asmundson *

Department of Psychology and Anxiety and Illness

Behaviour Laboratory, University of Regina,

3737 Wascana Parkway, Regina, SK, Canada S4S OA2

E-mail address: gordon.asmundson@uregina.ca

Joel Katz

Department of Psychology and School of Kinesiology and Health Science, York University, Toronto, Ont., Canada Department of Anesthesia and Pain Management, Toronto General Hospital and Mount Sinai Hospital, Toronto, Ont., Canada

Department of Anesthesia, University of Toronto, Toronto, Ont., Canada

* Corresponding author. Tel.: +1 306347 2415; fax: +1 306337 3275. 